\title{
Numerical Investigation of Pull-In Instability in a Micro-Switch MEMS Device through the Pseudo-Spectral Method
}

\author{
P. Di Maida and G. Bianchi \\ Dipartimento di Scienze e Metodi dell'Ingegneria (DISMI), Universitá degli Studi di Modena e Reggio Emilia, Via G. Amendola 2, \\ 42122 Reggio Emilia, Italy
}

Correspondence should be addressed to P. Di Maida; pietro.dimaida@unimore.it

Received 4 December 2015; Revised 10 May 2016; Accepted 5 October 2016

Academic Editor: Julius Kaplunov

Copyright (C) 2016 P. Di Maida and G. Bianchi. This is an open access article distributed under the Creative Commons Attribution License, which permits unrestricted use, distribution, and reproduction in any medium, provided the original work is properly cited.

\begin{abstract}
A pseudo-spectral approximation is presented to solve the problem of pull-in instability in a cantilever micro-switch. As well known, pull-in instability arises when the acting force reaches a critical threshold beyond which equilibrium is no longer possible. In particular, Coulomb electrostatic force is considered, although the method can be easily generalized to account for fringe as well as Casimir effects. A numerical comparison is presented between a pseudo-spectral and a Finite Element (FE) approximation of the problem, both methods employing the same number of degrees of freedom. It is shown that the pseudo-spectral method appears more effective in accurately approximating the behavior of the cantilever near its tip. This fact is crucial to capturing the threshold voltage on the verge of pull-in. Conversely, the FE approximation presents rapid successions of attracting/repulsing regions along the cantilever, which are not restricted to the near pull-in regime.
\end{abstract}

\section{Introduction}

Micro-Electro-Mechanical Systems (MEMS) form a rather diverse and inhomogeneous group of micro-devices aimed at sensing and actuating in a wide array of fields, ranging from mechanical or electronic engineering to chemistry or biology, from micro-mechanics to micro-machining [1-5]. The manufacturing technology is the common standground for such devices, which heavily relies on the different lithographic techniques borrowed from the technology of microelectronics. Indeed, MEMS devices are mostly obtained from a silicon substrate. It is observed that MEMS are really "systems" in the sense that they are often made up of several functional parts joint together in the device (like piezoand magneto-sensors [6]). Among MEMS, micro-switch forms a distinct set with great application potential, with special regard to phase shifters and Radio Frequency MEMS (RFMEMS). They are usually gathered in two groups, namely, capacitor and metal-air-metal switches. Besides, they are further divided according to the actuation method: electrostatic, electrothermal, magnetostatic, and piezoelectric among the most common. A study of magnetoelastic actuated microswitch is given in $[7,8]$ for the low-frequency asymptotic analysis of energy scavengers. In this paper, we focus attention on the pull-in instability of a capacitor micro-switch actuated by electrostatic Coulomb force. This particular application has received extensive attention in the literature, owing to the importance of pull-in induced failures in applications. A recent review on the subject can be found in [9]. A theoretical analysis of this problem within the static regime is provided in [10] and references therein. Pull-in voltage in cantilever MEMS have been considered in [11-14]. Failure mechanisms of MEMS include cracking [15-18], peeling of the cantilever [19-21], stiction to the substrate [22-24], and temperature $[25,26]$. Besides, micropolar theories are often preferred when dealing with micro- and nanodevices to incorporate the scale effect $[27,28]$. Spotlight is set on a pseudo-spectral approximation of the problem, which is compared with a Finite Element (FE) solution. Spectral methods belong to the family of Galerkin's (or Ritz's) methods [29]. Spectral methods are often divided into two groups, namely, pseudo-spectral or interpolating. The former group enforces the fulfillment of 


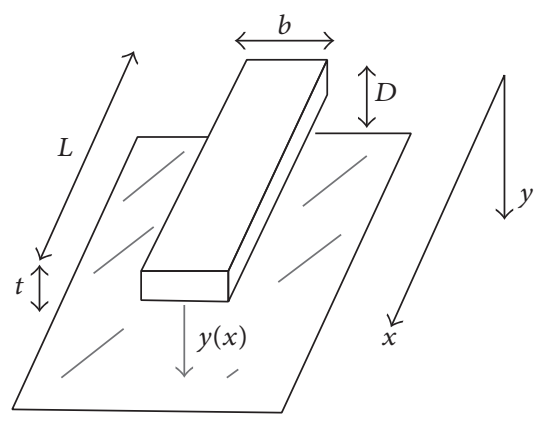

FIGURE 1: Micro-cantilever switch.

the differential operator at a set of points termed nodes (this is sometimes also named orthogonal collocation). For the latter group, wherein the Galerkin's method is properly placed, the expansion coefficients are obtained projecting the solution onto the basis set [30].

The paper is structured as follows: Section 2 sets forth the governing equations and the boundary condition for a cantilever. Section 3 introduces the pseudo-spectral method. A numerical comparison with the FE method is illustrated in Section 4. Finally, conclusions are drawn in Section 5.

\section{Governing Equations}

Let us consider a micro-cantilever switch device subjected to electrostatic attractive force (Figure 1). The micro-cantilever acts as one armor of a capacitor under the electric potential difference $V$. Let $t$ denote the distance between the capacitor armors. We consider a plane problem and introduce the transverse displacement $y(x)$ for the cantilever. Let us introduce the dimensionless variables

$$
\begin{aligned}
\xi & =\frac{x}{L}, \\
u & =\frac{y}{t} .
\end{aligned}
$$

Then the governing equation for the cantilever reads

$$
\frac{E I t}{L^{4}} \frac{\mathrm{d}^{4} u}{\mathrm{~d} \xi^{4}}=q_{c}+q_{e}
$$

where $q_{c}$ and $q_{e}$ are the Casimir and the electrostatic lineload, being

$$
q_{e}=\alpha \epsilon_{0} \frac{b V^{2}}{t^{2}(1-u)^{2}} .
$$

Here, $V$ stands for the electric potential difference acting between the capacitor armors (in the SI this is expressed in volt, i.e., $V=\mathrm{Nm} / \mathrm{C}$ where $\mathrm{N}$ stands for newton, $\mathrm{m}$ for meter, and $\mathrm{C}$ for electric charge, expressed in Coulomb), $b$ is the armors width, $\epsilon_{0}=8.854 \cdot 10^{-12} \mathrm{C}^{2} \mathrm{~N}^{-1} \mathrm{~m}^{-2}$ is the electric permittivity (in vacuum), and $\alpha$ is generally a function of $u$ which takes into account the fringe effect. For the sake of illustrating the method, we neglect the Casimir force contribution and assume $\alpha$ independent of $u$. Then, we can rewrite the governing equation (2) as

$$
u^{(i v)}=\frac{A}{(1-u)^{2}},
$$

where prime denotes differentiation with respect to $\xi$ and the following driving parameter is obtained:

$$
A=\alpha \epsilon_{0} \frac{b L^{4}}{t^{3} E I} V^{2} \geq 0 .
$$

Under the attractive electrostatic force, it is $0 \leq u(\xi) \leq u_{\max } \leq$ 1 and the boundary conditions (BCs) for the cantilever read

$$
u(0)=u^{\prime}(0)=u^{\prime \prime}(1)=u^{\prime \prime \prime}(1)=0 .
$$

Let us define $v(\xi)=1-u(\xi)$; thus (4) further reduces to

$$
v^{(i v)}=-\frac{A}{v^{2}},
$$

with $0 \leq v_{\min } \leq v(\xi) \leq 1$ and the BCs

$$
\begin{aligned}
& v(0)=1, \\
& v^{\prime}(0)=v^{\prime \prime}(1)=v^{\prime \prime \prime}(1)=0 .
\end{aligned}
$$

It is observed that, integrating and making use of the last BC, it may be deduced that

$$
v^{\prime \prime \prime}(\xi)=\int_{\xi}^{1} \frac{A}{v^{2}(\tau)} \mathrm{d} \tau,
$$

which shows that the shearing force is generally positive and it is zero only at $\xi=1$. The same argument may be applied to infer that $v^{\prime \prime}$ is generally negative, apart from the point $\xi=1$ where it is zero, and that $v^{\prime}$ is generally negative, although it vanishes at $\xi=0$. Consequently, $v$ is a monotonic decreasing function of $\xi$ and $v(1)=v_{\min }$. The nonlinear fourth order ODE (7) may be integrated once $[31, \$ 4.2 .1]$ to give

$$
2 v^{\prime} v^{\prime \prime \prime}-\left(v^{\prime \prime}\right)^{2}=\frac{2 A}{v}+\frac{4}{3} B
$$

It is observed that, in the case $B=0,(10)$ falls into the Emdem-Fowler class of nonlinear ODEs, which, in special cases, may admit closed form solutions [31, 32]. Evaluation at $\xi=1$ and making use of the BCs (8) give

$$
B=-\frac{3}{2} \frac{A}{v_{\min }},
$$

which shows that the situation $B=0$ is not relevant in this problem. Besides, it follows

$$
2 v^{\prime} v^{\prime \prime \prime}-\left(v^{\prime \prime}\right)^{2}=2 A\left(\frac{1}{v}-\frac{1}{v_{\min }}\right) \leq 0,
$$

and evaluation at $\xi=0$ lends

$$
\left[v^{\prime \prime}(0)\right]^{2}=2 A\left(\frac{1}{v_{\min }}-1\right) .
$$

Consideration of the sign for $v^{\prime}$ and $v^{\prime \prime \prime}$ yields the inequality

$$
v^{\prime \prime} \leq \sqrt{2 A\left(\frac{1}{v_{\min }}-\frac{1}{v}\right)} .
$$




\section{Pseudo-Spectral Method}

The governing equations (7) may be numerically solved through a pseudo-spectral approach [29, 33]. Accordingly, a $n$-degree polynomial function $p_{n}(\xi)$ is adopted to approximate the function $u(\xi)$ on the interval $[0,1]$. Since a collocation method is adopted, the polynomial will be uniquely determined enforcing (7) at some $n+1$ predetermined points (nodes). This procedure results in a system of nonlinear algebraic equations, which may be solved through standard methods, such as the iterative Newton method. The Jacobian of the system may be supplemented in closed form to the numerical equation solver.

Let $p_{n}(\xi) \in \mathscr{P}_{n}$ be the $n$-degree polynomial approximation of $v(\xi)$. The collocation set is defined through the first $n+1$ Gauss-Lobatto points

$$
\begin{aligned}
\mathrm{GL}_{n} & =\left\{\eta_{i} \text { such that } \eta_{0}=-1, \eta_{n}=+1, p_{n}^{\prime}\left(\eta_{i}\right)\right. \\
& =0, i=1, \ldots, n-1\},
\end{aligned}
$$

where $p_{n}$ stands for the $n$th degree Legendre polynomial. Equation (7), evaluated at the interior nodes $\eta_{i}$, yields the system of $(n-1)$ algebraic equations

$$
p_{n}^{2}\left(\eta_{i}\right) p_{n}^{(i v)}\left(\eta_{i}\right)+A=0, \quad i=1, \ldots, n-1,
$$

which is then supplemented with the boundary conditions (8)

$$
\begin{aligned}
& p_{n}\left(\eta_{0}\right)=1, \\
& p_{n}^{\prime}\left(\eta_{0}\right)=p_{n}^{\prime \prime}\left(\eta_{n}\right)=p_{n}^{\prime \prime \prime}\left(\eta_{n}\right)=0 .
\end{aligned}
$$

The problem is now rewritten in matrix form. To this aim, let the unknown column vector, the vector of the square, and the vector of the $m$ th derivative

$$
\begin{aligned}
\mathrm{x} & =\left[p_{n}\left(\eta_{i}\right)\right], \\
\mathrm{y} & =\left[p_{n}^{2}\left(\eta_{i}\right)\right], \\
\mathrm{x}^{(m)} & =\left[p_{n}^{(m)}\left(\eta_{i}\right)\right], \\
& \quad i=0, \ldots, n, m \in \mathbb{N} .
\end{aligned}
$$

Making use of the $(n+1) \times(n+1)$ derivative matrix $\mathrm{D}$ (see [33, Chap.7]) we have

$$
\begin{aligned}
& x^{\prime}=D x, \\
& x^{\prime \prime}=D D x=D^{2} x, \ldots
\end{aligned}
$$

and (16) and (17) may be rewritten through the derivative matrix as

$$
\mathrm{y} \widetilde{\mathrm{D}^{4}} \mathrm{x}+A \mathrm{Id}=\widetilde{\mathrm{o}}
$$

where Id is the identity matrix. Here, $\widetilde{D^{4}}$ is $D^{4}$ supplemented with the BCs (8); that is, the first row is set to zero apart from the first entry that is set to 1 ; the second, the last-but-one and

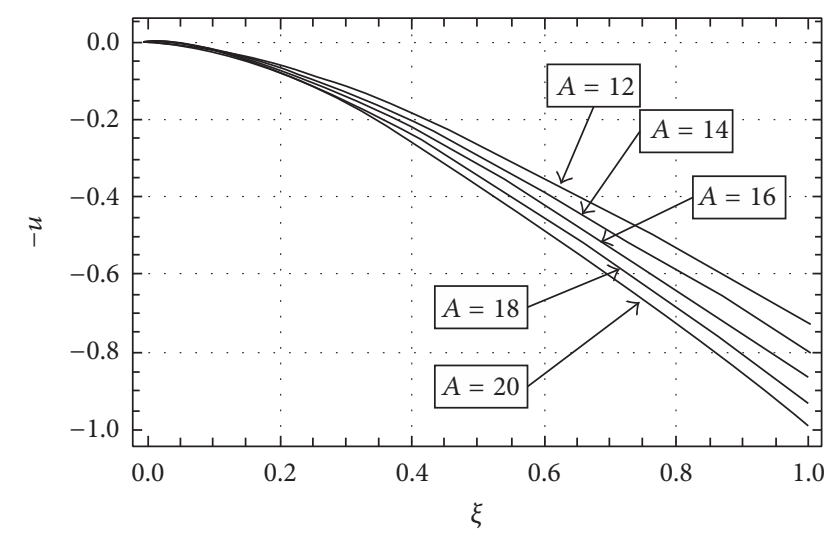

Figure 2: Displacement $u$ at $A=12,14,16,18$ and $A=20$.

the last rows are replaced by the first and the last rows of $D$, $D^{2}$, and $D^{3}$, respectively. Then we have

$$
\widetilde{\mathrm{D}^{4}}=\left[\begin{array}{cccc}
1 & 0 & \cdots & 0 \\
D_{0,0} & \cdots & & D_{0, n} \\
D_{2,0}^{4} & \cdots & & D_{2, n}^{4} \\
\vdots & \ddots & & \\
D_{n-2,0}^{4} & \cdots & & D_{n-2, n}^{4} \\
D_{n-1,0}^{2} & \cdots & & D_{n-1, n}^{2} \\
D_{n, 0}^{3} & D_{n, 1}^{3} & \cdots & D_{n, n}^{3}
\end{array}\right] .
$$

At the RHS of system (20) we have the zero vector o supplemented with the BCs, namely,

$$
\widetilde{\mathrm{o}}=\left[\begin{array}{c}
1 \\
0 \\
\vdots \\
0
\end{array}\right] .
$$

\section{Numerical Solution}

In this section, the pseudo-spectral approximation is compared with a Finite Element solution, both methods employing the same number of degrees of freedom, which corresponds to $n=20$ for the order of the interpolating polynomial. For the FE solution, we introduce 10 nodes, each endowed with 2 degrees of freedom, that is, one translational and the other rotational. In Figure 2, the system of algebraic equations (20) is solved for different values of the driving parameter (voltage difference) $A$ with the FE method. It clearly appears that, for $A=20$, the cantilever dimensionless tip deflection is very close to 1 ; that is, the cantilever is at the verge of pull-in instability. Figure 3 shows the product $-v^{(i v)} v^{2}$, which should be constant along the cantilever and equal to $A$, for the FE approximation. It is evident that the quality of the numerical solution rapidly deteriorates near the cantilever tip, which is exactly where best accuracy 


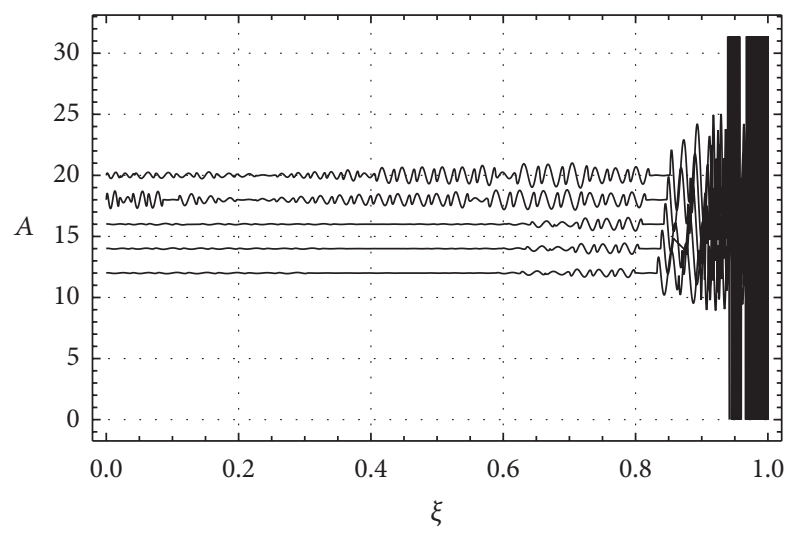

FIgURE 3: Plot of $-v^{2} v^{(i v)}$ versus $\xi$ according to the FE approximation: this product should return the constant $A$.

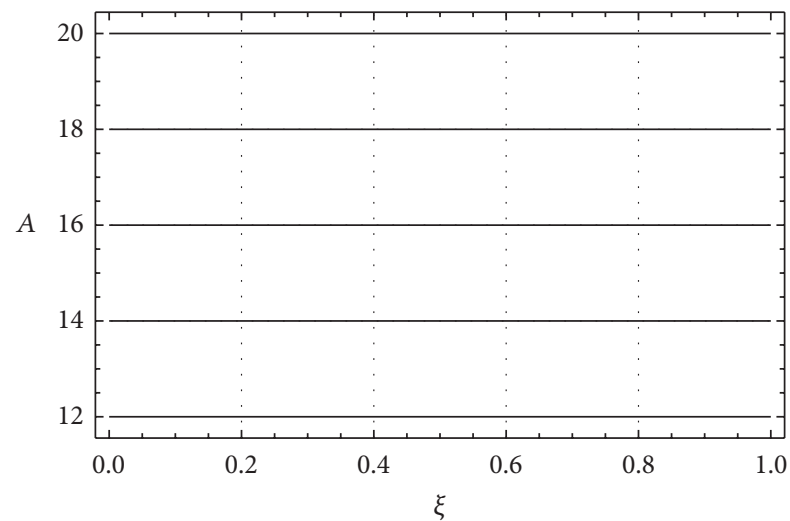

FIGURE 4: Plot of $-v^{2} v^{(i v)}$ versus $\xi$ according to the pseudo-spectral approximation: this product should return the constant $A$.

is demanded to effectively capture the pull-in threshold. Conversely, Figure 4 plots the product $-v^{(i v)} v^{2}$ for the pseudospectral approximation. The comparison between the two plots is a striking example of the effectiveness of this method in this kind of nonlinear problems. Figure 5 plots the slope, bending moment and shearing force along the cantilever beam, and it illustrates that BCs are well captured by the numerical solution, either FE or pseudo-spectral. The plot is obtained by successive differentiation of the displacement field and it is readily available for the spectral method, where polynomial functions are employed. Conversely, obtaining the corresponding curves for the FE approximation needs some extra care for curve fitting of the nodal displacement is first applied, which is then successively differentiated. Figure 6 plots the applied line load density $v^{(i v)}$ for the FE solution near pull-in, which corresponds to (the negative of) the electrostatic Coulomb force $-q_{e}$. It is remarkable that the electrostatic force appears highly oscillatory (note that curve-fitting is employed to get a continuous plot), and it attains unphysical negative values. As a comparison, Figure 7 describes the same behavior for $A=12$, that is well below the threshold value for pull-in. It appears that the electrostatic force is rather poorly approximated by the

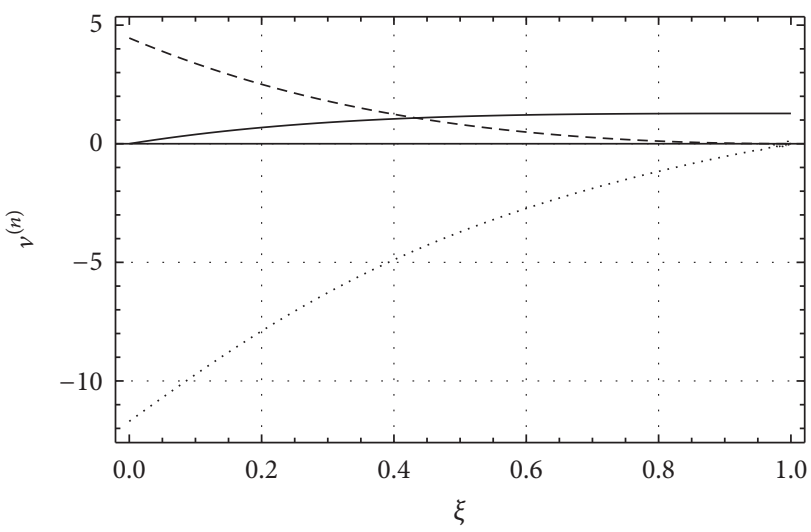

FIGURE 5: Slope (solid), bending moment (dashed), and shearing force (dotted) near pull-in. Graphs are indistinguishable between the pseudo-spectral and the FE solution.

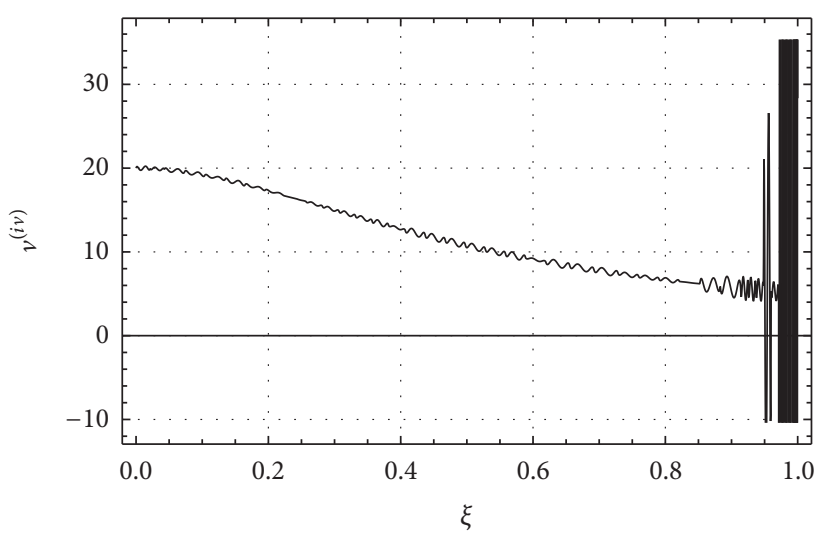

Figure 6: Line load density $q_{e}$ on the cantilever beam near pull-in according to the FE approximation $(A=20)$.

method, even far from instability. On the contrary, Figures 8 and 9 illustrate the same graphs as obtained from the pseudo-spectral method. The smoothness of the solution is remarkable. The reason for the superior performance of the pseudo-spectral approximation seems to lie in the fact that it is more robust in dealing with little deviations of the cantilever tip displacement on the verge of contact. Indeed, it is well known that pseudo-spectral approximation guarantees high precision and exponential convergence (under suitable assumptions, see [29]), and this feature proves important in smoothly approximating the highly nonlinear behavior of the electrostatic force.

\section{Conclusions}

In this paper, the pseudo-spectral method is adopted to numerically solve the problem of pull-in instability in a cantilever beam. The beam constitutes one armor of a capacitor, the other armor being represented by a grounded flat surface. Although only Coulomb electrostatic force is considered, the method is easily extended to deal with the fringe effect and 


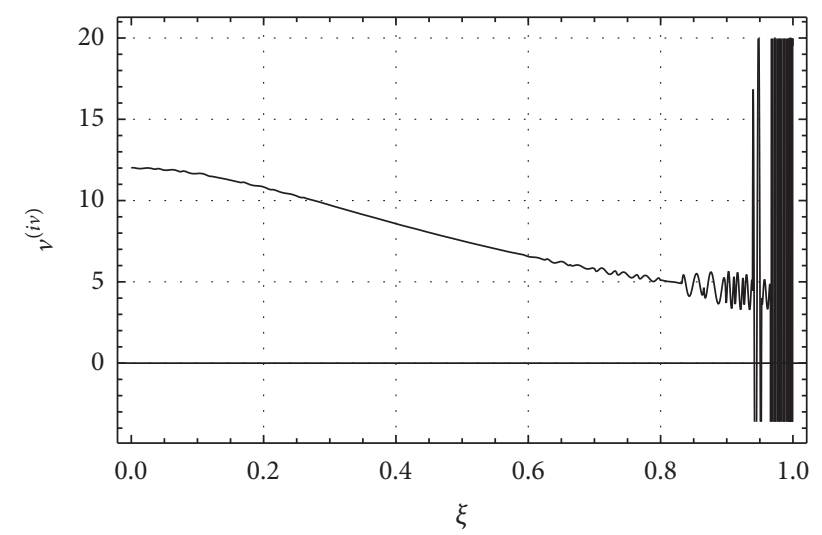

Figure 7: Line load density $q_{e}$ on the cantilever beam near pull-in according to the FE approximation $(A=12)$.

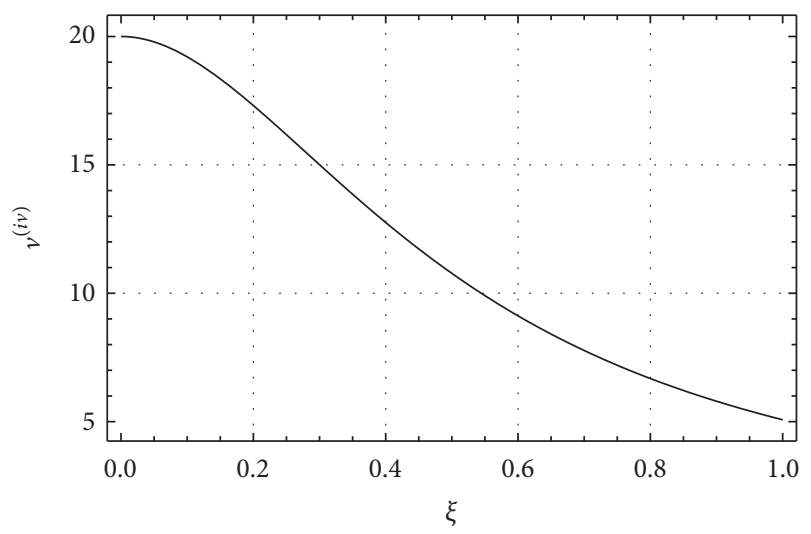

Figure 8: Line load density $q_{e}$ on the cantilever beam near pull-in according to the pseudo-spectral approximation $(A=20)$.

the Casimir force. Results may be especially relevant for cantilever micro-switches in MEMS. It is shown that the pseudospectral method compares very favorably with an equivalent Finite Element approximation, equivalency being constituted by an equal number of degrees of freedom in the methods. In particular, good approximation for the cantilever deflection near its tip is crucial to capturing the threshold voltage on the verge of pull-in. Indeed, poor approximation leads to very unphysical oscillatory attraction/repulsion forces along the cantilever. It is further shown that the oscillatory behavior is not restricted to the near pull-in regime. Finally, it is emphasized that both methods exactly satisfy the boundary conditions (BCs). It is remarked that the present analysis can be extended to incorporate functionally graded cantilevers [34-36] and beam-plates $[37,38]$ or to include viscoelastic effects [39-42].

\section{Competing Interests}

The authors declare that they have no competing interests.

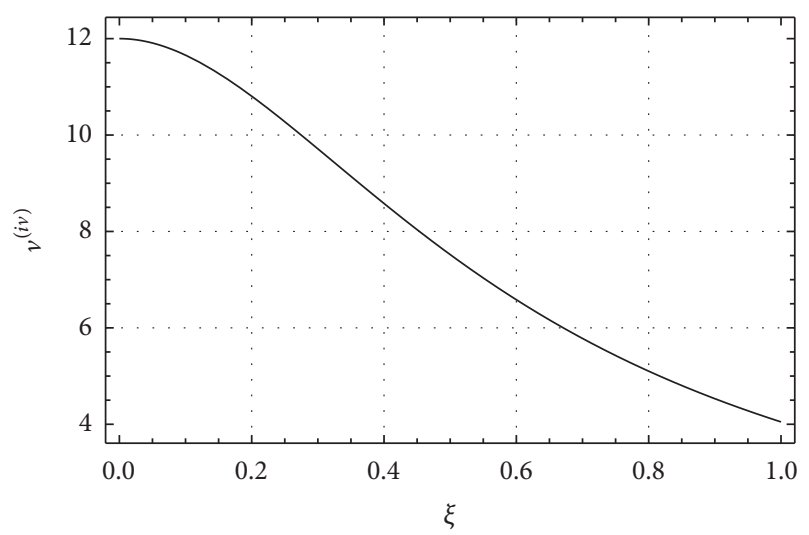

FIgURE 9: Line load density $q_{e}$ on the cantilever beam near pull-in according to the pseudo-spectral approximation $(A=12)$.

\section{References}

[1] V. A. Salomoni, C. E. Majorana, G. M. Giannuzzi, and A. Miliozzi, "Thermal-fluid flow within innovative heat storage concrete systems for solar power plants," International Journal of Numerical Methods for Heat and Fluid Flow, vol. 18, no. 7-8, pp. 969-999, 2008.

[2] A. Nobili, L. Lanzoni, and A. M. Tarantino, "Experimental investigation and monitoring of a polypropylene-based fiber reinforced concrete road pavement," Construction and Building Materials, vol. 47, pp. 888-895, 2013.

[3] V. A. Salomoni, C. E. Majorana, B. Pomaro, G. Xotta, and F. Gramegna, "Macroscale and mesoscale analysis of concrete as a multiphase material for biological shields against nuclear radiation," International Journal for Numerical and Analytical Methods in Geomechanics, vol. 38, no. 5, pp. 518-535, 2014.

[4] G. Dinelli, G. Belz, C. E. Majorana, and B. A. Schrefler, "Experimental investigation on the use of fly ash for lightweight precast structural elements," Materials and Structures, vol. 29, no. 194, pp. 632-638, 1996.

[5] P. Bisegna and R. Luciano, "Bounds on the overall properties of composites with debonded frictionless interfaces," Mechanics of Materials, vol. 28, no. 1-4, pp. 23-32, 1998.

[6] A. Nobili and A. M. Tarantino, "Magnetostriction of a hard ferromagnetic and elastic thin-film structure," Mathematics and Mechanics of Solids, vol. 13, no. 2, pp. 95-123, 2008.

[7] A. Kudaibergenov, A. Nobili, and L. Prikazchikova, "On lowfrequency vibrations of a composite string with contrast properties for energy scavenging fabric devices," Journal of Mechanics of Materials and Structures, vol. 11, no. 3, pp. 231-243, 2016.

[8] J. Kaplunov and A. Nobili, "Multi-parametric analysis of strongly inhomogeneous periodic waveguideswith internal cutoff frequencies," Mathematical Methods in the Applied Sciences, 2016.

[9] W.-M. Zhang, H. Yan, Z.-K. Peng, and G. Meng, "Electrostatic pull-in instability in MEMS/NEMS: a review," Sensors and Actuators A: Physical, vol. 214, pp. 187-218, 2014.

[10] Y. Zhang and Y.-P. Zhao, "Static study of cantilever beam stiction under electrostatic force influence," Acta Mechanica Solida Sinica, vol. 17, no. 2, pp. 104-112, 2004.

[11] L. C. Wei, A. B. Mohammad, and N. M. Kassim, "Analytical modeling for determination of pull-in voltage for an electrostatic actuated MEMS cantilever beam," in Proceedings of the 
5th IEEE International Conference on Semiconductor Electronics (ICSE '02), pp. 233-238, IEEE, Penang, Malaysia, December 2002.

[12] S. Chowdhury, M. Ahmadi, and W. C. Miller, "A closed-form model for the pull-in voltage of electrostatically actuated cantilever beams," Journal of Micromechanics and Microengineering, vol. 15, no. 4, pp. 756-763, 2005.

[13] H. Sadeghian, G. Rezazadeh, and P. M. Osterberg, "Application of the generalized differential quadrature method to the study of pull-in phenomena of MEMS switches," Journal of Microelectromechanical Systems, vol. 16, no. 6, pp. 1334-1340, 2007.

[14] A. Ramezani, A. Alasty, and J. Akbari, "Closed-form solutions of the pull-in instability in nano-cantilevers under electrostatic and intermolecular surface forces," International Journal of Solids and Structures, vol. 44, no. 14-15, pp. 4925-4941, 2007.

[15] A. M. Tarantino, "On the finite motions generated by a mode I propagating crack," Journal of Elasticity, vol. 57, no. 2, pp. 85103, 1999.

[16] A. M. Tarantino, "Nonlinear fracture mechanics for an elastic Bell material," The Quarterly Journal of Mechanics and Applied Mathematics, vol. 50, no. 3, pp. 435-456, 1997.

[17] R. Luciano and J. R. Willis, "Hashin-Shtrikman based FE analysis of the elastic behaviour of finite random composite bodies," International Journal of Fracture, vol. 137, no. 1-4, pp. 261-273, 2006.

[18] A. Nobili, E. Radi, and L. Lanzoni, "A cracked infinite Kirchhoff plate supported by a two-parameter elastic foundation," Journal of the European Ceramic Society, vol. 34, no. 11, pp. 2737-2744, 2014.

[19] L. Lanzoni and E. Radi, "Thermally induced deformations in a partially coated elastic layer," International Journal of Solids and Structures, vol. 46, no. 6, pp. 1402-1412, 2009.

[20] V. Guidi, L. Lanzoni, and A. Mazzolari, "Patterning and modeling of mechanically bent silicon plates deformed through coactive stresses," Thin Solid Films, vol. 520, no. 3, pp. 1074-1079, 2011.

[21] N. Tullini, A. Tralli, and L. Lanzoni, "Intefacial shear stress analysis of bar and thin film bonded to 2D elastic substrate using a coupled FE-BIE method," Finite Elements in Analysis and Design, vol. 55, pp. 42-51, 2012.

[22] C. H. Mastrangelo, Suppression of Stiction in MEMS, vol. 605 of MRS Proceedings, Cambridge University Press, Cambridge, UK, 1999.

[23] W. Merlijn Van Spengen, R. Puers, and I. De Wolf, "A physical model to predict stiction in MEMS," Journal of Micromechanics and Microengineering, vol. 12, no. 5, pp. 702-713, 2002.

[24] Z. Yapu, "Stiction and anti-stiction in MEMS and NEMS," Acta Mechanica Sinica, vol. 19, no. 1, pp. 1-10, 2003.

[25] G. Xotta, G. Mazzucco, V. A. Salomoni, C. E. Majorana, and K. J. Willam, "Composite behavior of concrete materials under high temperatures," International Journal of Solids and Structures, vol. 64, pp. 86-99, 2015.

[26] F. Marotti de Sciarra and M. Salerno, "On thermodynamic functions in thermoelasticity without energy dissipation," European Journal of Mechanics-A: Solids, vol. 46, pp. 84-95, 2014.

[27] A. Nobili, "On the generalization of the Timoshenko beam model based on the micropolar linear theory: static case," Mathematical Problems in Engineering, vol. 2015, Article ID 914357, 8 pages, 2015.

[28] G. Napoli and A. Nobili, "Mechanically induced HelfrichHurault effect in lamellar systems," Physical Review E, vol. 80, no. 3, Article ID 031710, 2009.
[29] J. P. Boyd, Chebyshev and Fourier Spectral Methods, Dover Publications, 2nd edition, 2000.

[30] F. Greco and R. Luciano, "A theoretical and numerical stability analysis for composite micro-structures by using homogenization theory," Composites-Part B: Engineering, vol. 42, no. 3, pp. 382-401, 2011.

[31] A. D. Polyanin and V. F. Zaitsev, Handbook of Exact Solutions for Ordinary Differential Equations, CRC Press, Boca Raton, Fla, USA, 1st edition, 1995.

[32] P. L. Sachdev, Nonlinear Ordinary Differential Equations and Their Applications, Marcel Dekker, 1991.

[33] D. Funaro, Polynomial Approximation of Differential Equations, vol. 8 of Lecture Notes in Physics, Springer, 1992.

[34] R. Barretta, L. Feo, R. Luciano, and F. Marotti de Sciarra, "Variational formulations for functionally graded nonlocal Bernoulli-Euler nanobeams," Composite Structures, vol. 129, pp. 80-89, 2015.

[35] R. Barretta, L. Feo, R. Luciano, and F. Marotti de Sciarra, "A gradient Eringen model for functionally graded nanorods," Composite Structures, vol. 131, pp. 1124-1131, 2015.

[36] R. Barretta, L. Feo, and R. Luciano, "Some closed-form solutions of functionally graded beams undergoing nonuniform torsion," Composite Structures, vol. 123, pp. 132-136, 2015.

[37] R. Barretta and R. Luciano, "Exact solutions of isotropic viscoelastic functionally graded Kirchhoff plates," Composite Structures, vol. 118, no. 1, pp. 448-454, 2014.

[38] A. Apuzzo, R. Barretta, and R. Luciano, "Some analytical solutions of functionally graded Kirchhoff plates," Composites Part B: Engineering, vol. 68, pp. 266-269, 2015.

[39] L. Dezi, G. Menditto, and A. M. Tarantino, "Homogeneous structures subjected to repeated structural system changes," Journal of Engineering Mechanics, vol. 116, no. 8, pp. 1723-1732, 1990.

[40] L. Dezi and A. M. Tarantino, "Time-dependent analysis of concrete structures with a variable structural system," $A C I$ Materials Journal, vol. 88, no. 3, pp. 320-324, 1991.

[41] L. Dezi, G. Menditto, and A. M. Tarantino, "Viscoelastic heterogeneous structures with variable structural system," Journal of Engineering Mechanics, vol. 119, no. 2, pp. 238-250, 1993.

[42] R. Barretta, L. Feo, and R. Luciano, "Torsion of functionally graded nonlocal viscoelastic circular nanobeams," Composites Part B: Engineering, vol. 72, pp. 217-222, 2015. 


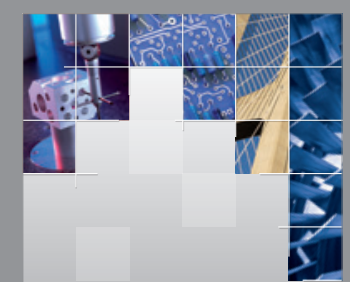

\section{Enfincering}
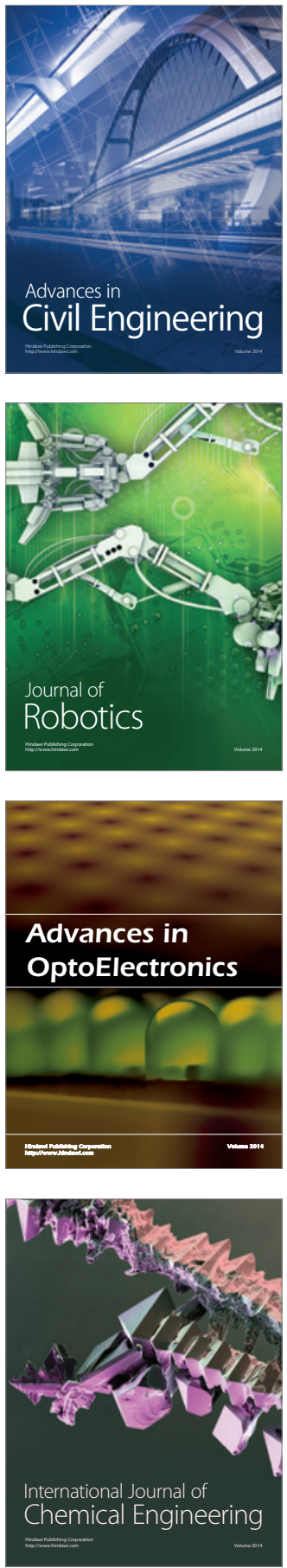

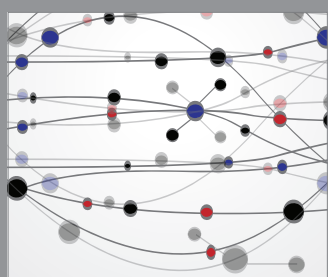

The Scientific World Journal

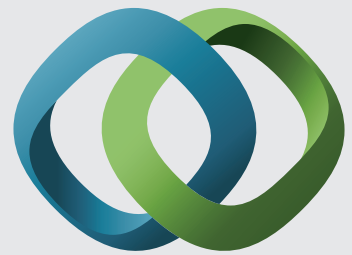

\section{Hindawi}

Submit your manuscripts at

http://www.hindawi.com
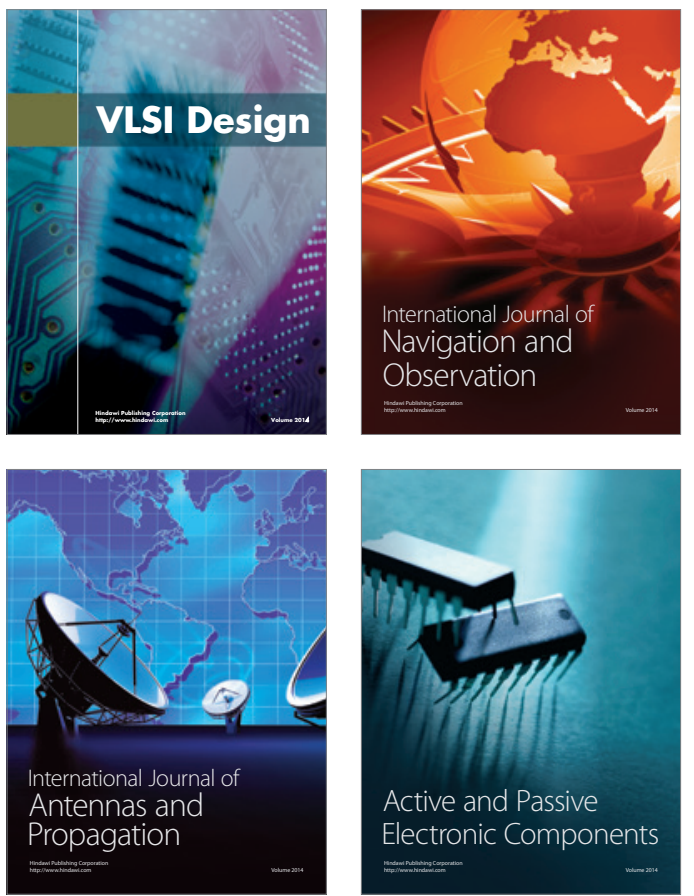
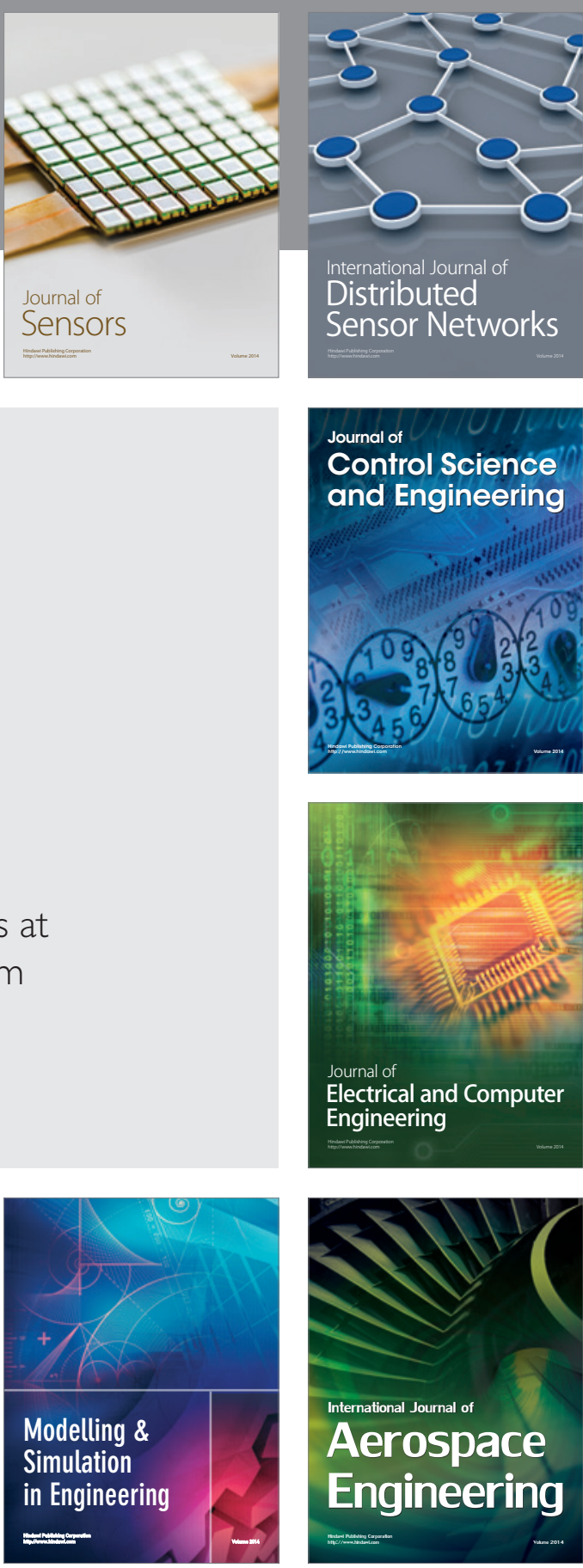

International Journal of

Distributed

Sensor Networks

Journal of

Control Science

and Engineering
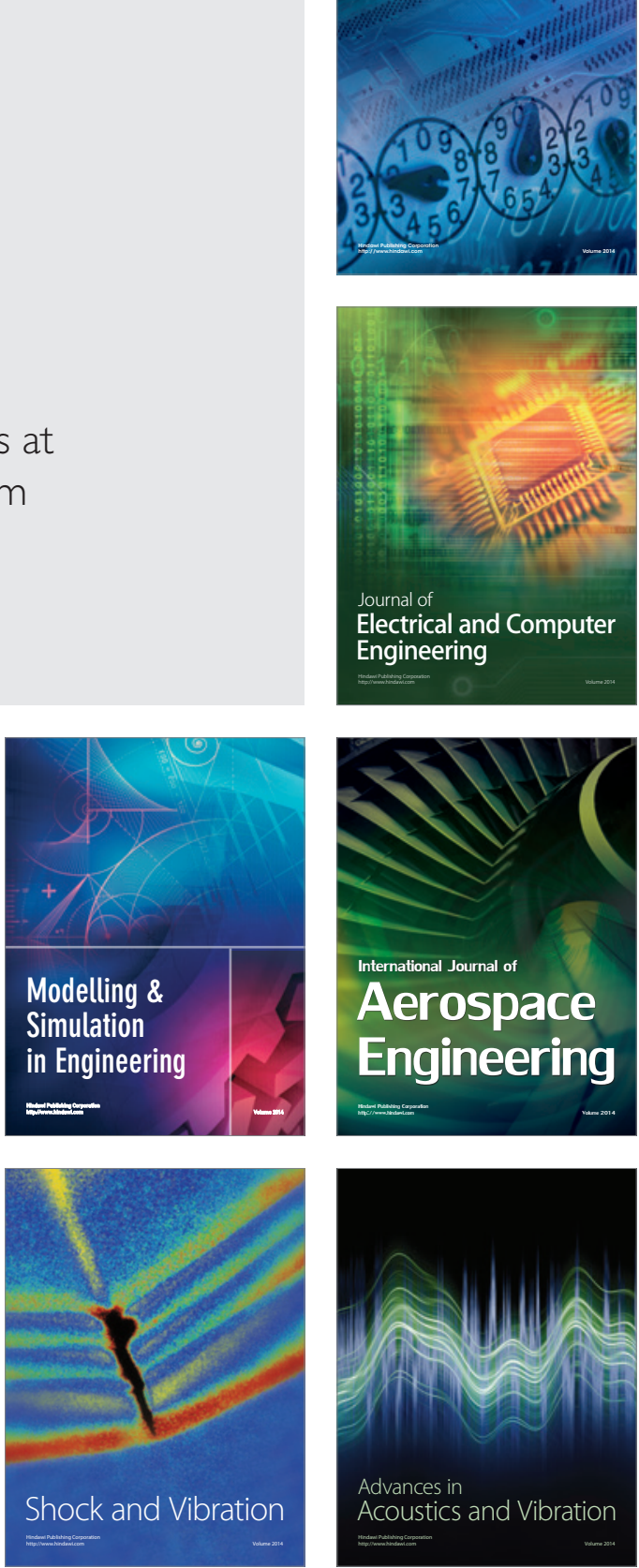